\title{
Maximizing Bidirectional Green Waves for Major Road Axes
}

Christian Liebchen (TH Wildau)

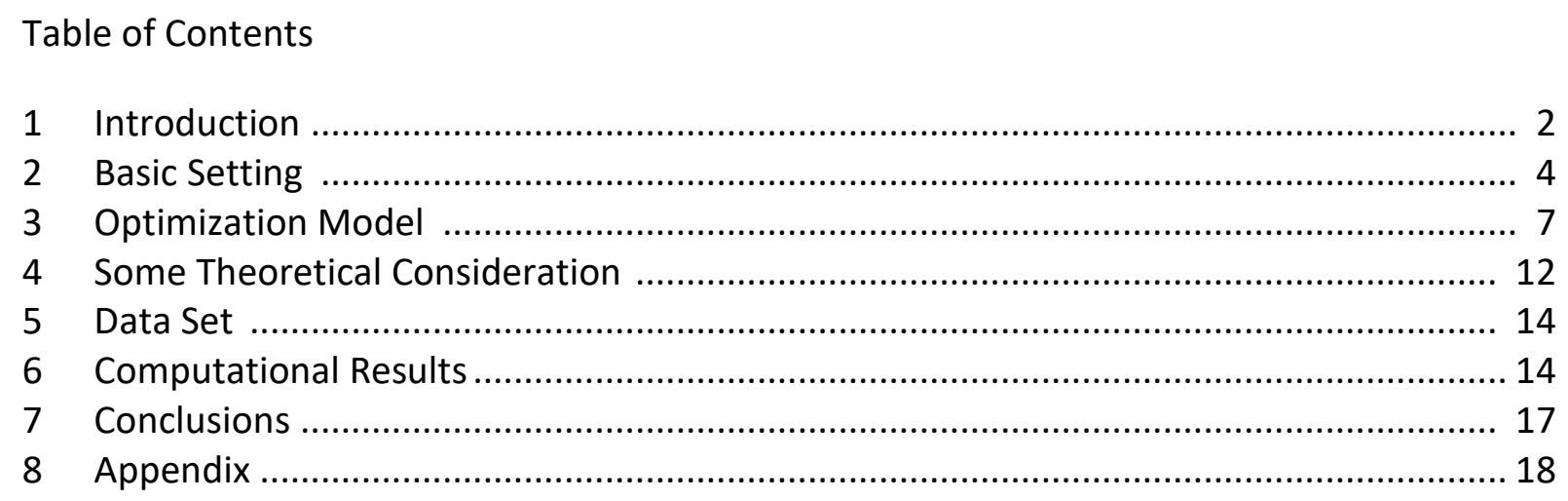

\section{PREPRINT}

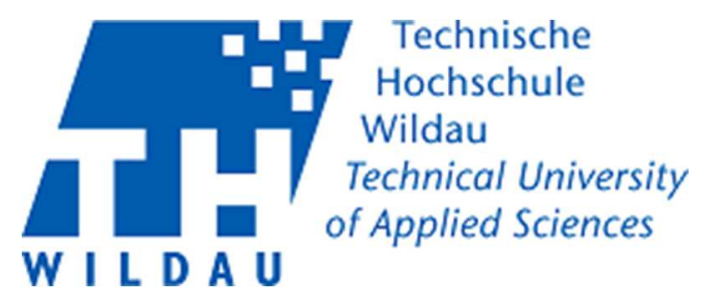

Submitted

Licence: CC-BY-NC-ND 


\title{
Maximizing Bidirectional Green Waves for Major Road Axes
}

\author{
Christian Liebchen
}

Technische Hochschule Wildau

Hochschulring 1

15745 Wildau, Germany

liebchen@th-wildau.de

\section{Abstract}

Both, from an environmental perspective and with respect to road traffic flow quality, planning so-called green waves along major road axes is a well-established target for traffic engineers. For one-way road axes (e.g. the Avenues in Manhattan), this is a trivial downstream task. For bidirectional arterials, the well-known necessary condition for establishing a green wave in both direction is that the driving times between two subsequent crossings must be an integer multiple of half of the cycle time of the signal programs at the nodes.

In this paper, we propose an integer linear optimization model to establish fixed-time green waves in both directions that are as long and as wide as possible, even in the situation where the driving time condition is not fulfilled. In particular, we are considering an arterial along whose nodes separate left-turn signal groups are realized.

In our computational results, we provide examples which illustrate that allowing for selecting among different strategies for the left-turn phases is beneficial. Moreover, we show that there is always a solution with green waves in both directions that are as long and as wide as possible, where absolute priority is put on just one direction. Only when considering prioritized parts of a green band (e.g. some first few seconds), then an ideal green wave into one direction can provide suboptimal quality compared to optimizing both directions together.

\section{Keywords}

traffic light coordination; synchronization; phase sequencing; green waves; integer programming;

\section{Introduction}

In particular, during times that show relatively high traffic volumes in various directions and dense networks with relatively short distances between junctions, fixed-time controlled signal programs at street crossings are likely to be a matter of choice (Foresite, 2021). When considering one isolated junction (or node), there are essentially the following decisions to be taken:

- definition of the phases (signal groups that are showing green at the same time),

- sequence of the phases, and

- lengths of the phases.

When counting any intermediate times as "phases", too, then the sum of the lengths of all phases yields the so-called cycle length, after which the very same signal program is going to repeat in a fixed-time control. In practice, due to the coordination between neighboring junctions, a common cycle length might be given first, and then the lengths of the phases are computed as fractions of the cycle length (red-green split).

As long as only one direction of traffic flow has to be considered along an arterial, the coordination of a sequence of junctions is trivial, because one only has to follow the movement of the first and last cars that pass at green (and thus delimit the so-called platoon 
of all cars in between) and propagate their passing times to the subsequent junctions. This may be a viable procedure if a one-way street is present, or at least if one of the two directions of the traffic flow can almost be neglected due to a very small traffic volume.

In the more general case, i.e., if both directions deserve attention, things are becoming more complicated. In Figure 1, it can be observed immediately, that a so-called "green wave" (illustrated by a green band) can be established for both directions, if the driving time between two subsequent nodes equals half of the common cycle length of the nodes. In more general, this is still true when the driving time equals some integer multiple of half of the common cycle length.

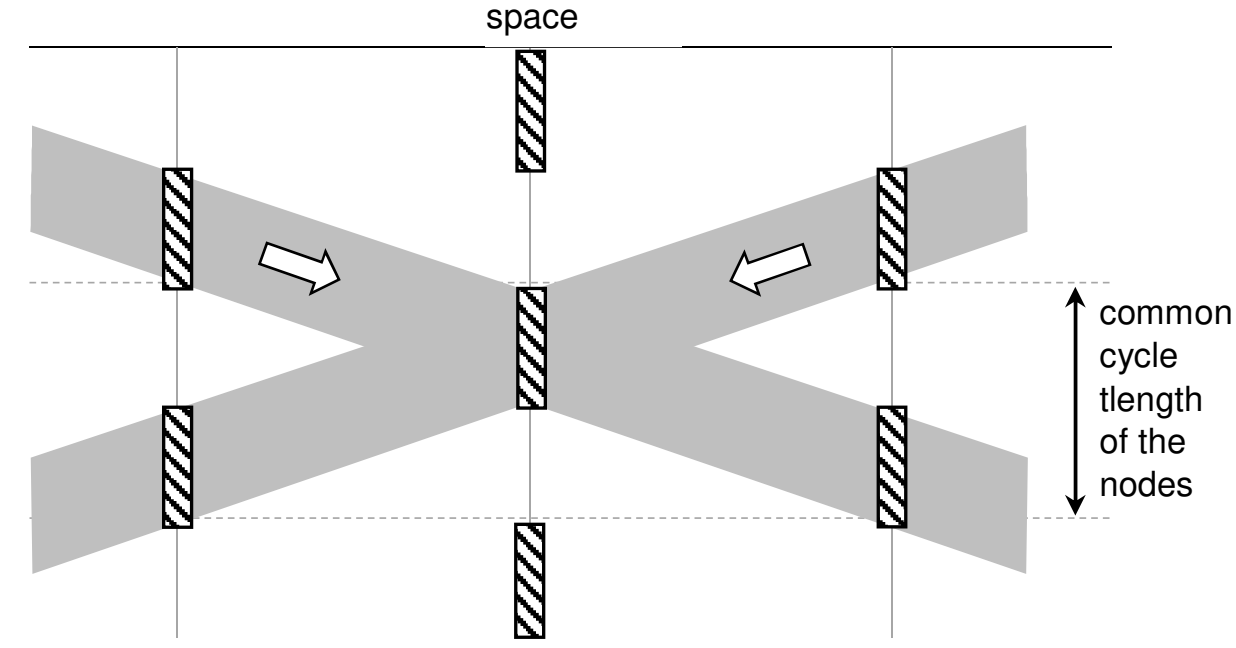

$\mathbf{D}$ green interval along the major road axis at some node

Figure 1 Three subsequent junctions along aan arterial. $A$ "green wave" (the grey bars represent one greenband for each direction) can be established into both directions, because the driving time between two subsequent junctions is (an integer multiple of) half the common cycle length of the signal programs at the junctions.

Yet, along real-world streets - unless these were planned from scratch instead of following developing urban structures - this particular requirement on the driving times is not always met. By simply coordinating (or synchronizing) the relative offsets (or shifts) of the signal programs of subsequent junctions, in general it is of course not possible to establish green waves into both directions. This is why in this paper we are going not only to consider the coordination of predefined signal programs, but also allow for changes in the signal programs at the junctions. In more detail, we are going to define appropriate sequences of the phases, and even select among a predefined small set of alternative phases.

In the literature, there can be found applications of mathematical optimization for the coordination of traffic lights, not only along arterials, but even for intermeshed street networks (Serafini and Ukovich, 1989; Hassin 1996). Along this line of research, even sophisticated objective functions to measure the waiting times of arriving cars appropriately have been designed and exploited during practical computations (Wünsch, 2008, in particular Section 1.3.5). Yet, for just one given bi-directional arterial, this approach has to remain limited, when the signal programs at the nodes have to remain fixed, within this conceptual framework.

But there are also other methods than mathematical optimization that are applied in the practice of traffic engineering. These include heuristic methods such as genetic algorithms and simulated annealing algorithms (see e.g. Binning et al., 2010). Their solution space indeed can go beyond the pure inter-node synchronization of pre-defined fixed signal 
programs, but make adjustments to the intra-node sequences and even lengths of the phases. However, it is within the nature of these algorithmic approaches that whatever objective function is going to be considered, they are not designed to prove that any solution that they compute, could be optimal, i.e., minimize the waiting times and/or stops of all vehicles. Zhang et al. (2015) consider similar decisions in terms of phase sequencing along two arterials, and compute their solutions by the means of integer linear optimization models.

With this background, the contribution of this paper lies in the combination of

- sticking to mathematical optimization methods,

- broadening their scope to decisions of phase sequencing and even partial selection among a limited number of pre-defined alternative phases, at the price of

- limiting the use case to arterials (with separate left-turn signal groups) instead of scheduling general road networks.

In addition, our computational results show that allowing for selecting among different strategies for the left-turn phases is beneficial. Moreover, we show that there is always a solution with green waves in both directions that are as long and as wide as possible, where absolute priority is put on just one direction. Only when considering prioritized parts of a green band, e.g. because we want prevent cars that stopped at red from having to stop right at the next junction again, then an ideal green wave into one direction can provide suboptimal quality compared to optimizing both directions together. The mathematical model that is presented in this paper shall be understood merely as an instrument to enable such findings from a traffic engineering point of view. In contrast, tuning the performance of solving the integer linear programs, e.g. for longer arterials and/r even intermeshed networks, is left to possible future merely mathematical contributions.

To summarize, in the notation of Eom and Kim (2020), the setting considered in this paper has the following properties:

- Network type: A (Arterial network)

- Real-time strategies: Fixed-time

- Objectives: TS (Total vehicle stops minimization, being approximated here)

- Cycle length: F (Fixed cycle length)

- Green phase duration: M, X (green phase duration limited by the same minimum and maximum)

- Phase sequence: $\mathrm{S}$ (Phase sequence is selected among phase groups)

\section{Basic Setting}

We are considering a major road axis (arterial) along which the left-turn traffic is secured by separate signal groups. For instance, in Berlin, Germany, this scenario applies to several radial axes in the eastern part of the city, where tramway tracks are located in between the lanes for the inbound and outbound traffic.

A well-established goal for the coordination of subsequent traffic lights along a main street is defined in the German street capacity manual (HBS Teil S, FGSV 2015):

"The quality of the traffic flow in a sequence of coordinated traffic lights can be assessed by a co-called coordination measure k. [...] This coordination measure $\mathrm{k}$ 
describes the mean portion of nodes with traffic lights in the coordinated sequence, which can be passed by the cars of the coordinated traffic stream without stop."1

We approximate this "official" goal by maximizing the portion of the entire green interval of an upstream node that arrives at the subsequent node downstream at green. We are aware that there is indeed a certain difference between the actual goal and our approximating objective. A major reason for this is that the probability for a car to pass the green interval at some node is not distributed uniformly. Rather, the first seconds of a green interval collect any car that "stranded" during the red phase, be it due to a lack of coordination or because the car just entered the major road axis from a crossing street or by just starting from its parking lot.

Nevertheless, there are two major reasons why we are not considering these effects accurately. First, this would result in a much more challenging mathematical optimization model, because we would have to follow paths that are starting at each of the nodes of the major road axis. Then, we have to detect by appropriate variables in the model down to which subsequent nodes which part of the green interval of the starting node of the path reaches the first, second, third etc. node downstream without being cut off, see Figure 2 . The decisions and relevant computations hence loose much of their primarily local nature, as it is a feature of the model that we are proposing. Second, in order to incorporate incoming traffic from crossing streets, the model should be fed with data on these traffic volumes relatively to the volume along the major road axis.

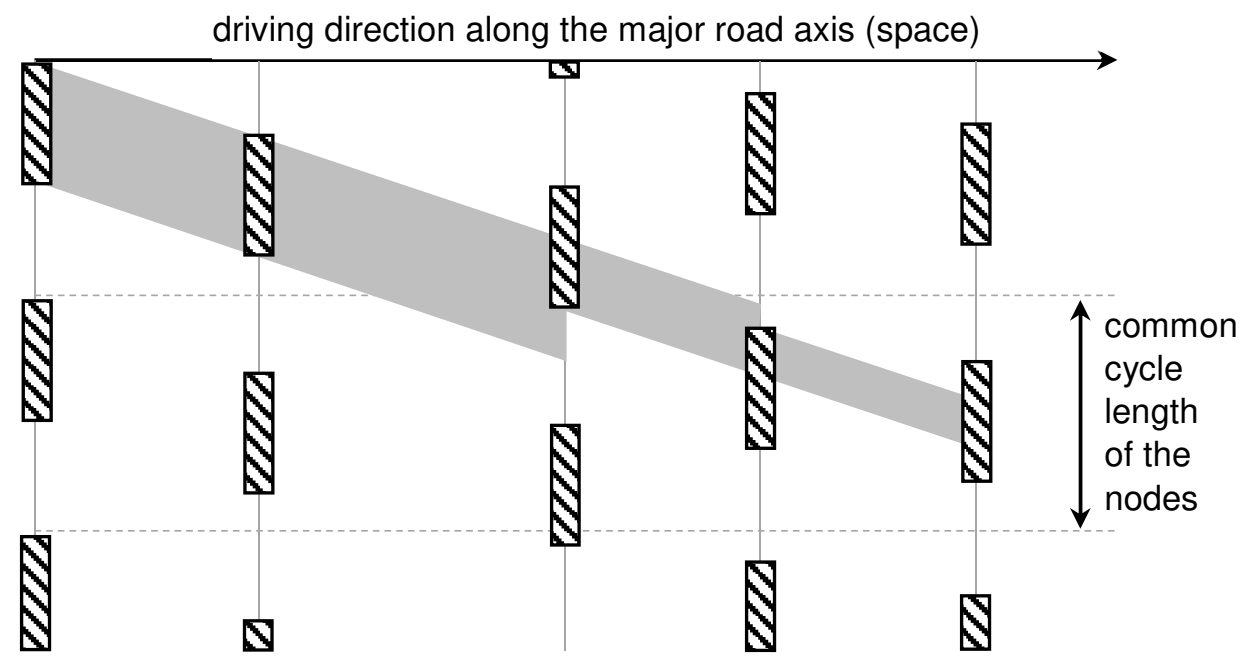

$\mathbf{D}$ green interval along the major road axis at some node

Figure 2 Time-space diagram in order to illustrate the different portions of an initial green interval at the left-most node ending at the third, last, and second next nodes downstream, respectively.

In order to obtain solutions that are as good as possible, at the nodes we do not only shift signal programs that are defined to be fixed a priori. Rather, in addition to such a pure coordination, we also include into our mixed-integer linear mathematical optimization model the degrees of freedom to modify the sequence of pre-defined phases, and even to select among a limited number of candidate phases.

1 „Die Verkehrsqualität einer Folge von koordinierten Lichtsignalanlagen kann durch das Koordinierungsmaß k beurteilt werden. [...] Das Koordinierungsmaß k beschreibt den mittleren Anteil der Knotenpunkte mit Lichtsignalanlagen in der koordinierten Folge, die von den Fahrzeugen im koordinierten Verkehrsstrom ohne Halt passiert werden kann." 
In the case where separate left-turn signal groups are implemented at a junction, there are the following four relevant basic phases for the through-traffic along the arterial and the separate left-turn traffic, as they are collected in Figure 3.

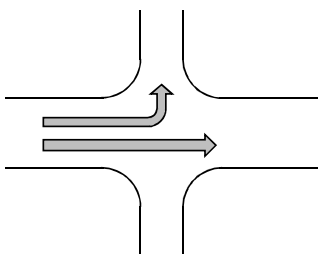

(a)

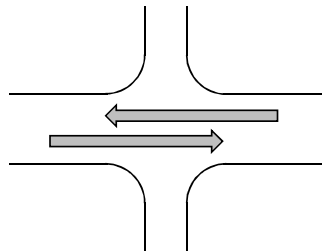

(b)

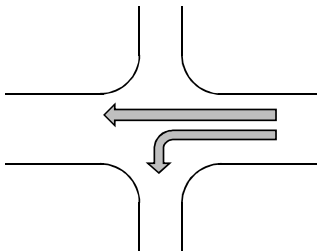

(c)

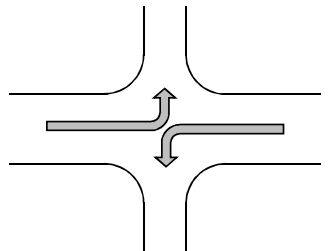

(d)

Figure 3 There are four relevant basic phases for the through-traffic along the arterial together with separate leftturn signal groups. The phases for the crossing traffic are not displayed, but are assumed to be connected (see also Zhang et al., 2015, Flg. 2).

In our experimental setup, w.I.o.g. we are going to consider a cycle time of $90 \mathrm{~s}$ for all the nodes along the major road axis (see also DOT, 2021, Table 6-1). In order to realize green durations of $50 \mathrm{~s}$ for the straight traffic and of $15 \mathrm{~s}$ for the left-turn traffic, the following four combinations and sequences of the four relevant basic phases in Table 1 are feasible:

Table 1 Overview of the four relevant sequences of the four relevant basic phases

\begin{tabular}{|l|l|l|l|l|l|}
\hline Sequence & First phase & $\begin{array}{l}\text { Second } \\
\text { Phase }\end{array}$ & Third Phase & Sum straight & $\begin{array}{l}\text { Sum left- } \\
\text { turn }\end{array}$ \\
\hline I & (a) $15 \mathrm{~s}$ & (b) $35 \mathrm{~s}$ & (c) $15 \mathrm{~s}$ & $50 \mathrm{~s}$ & $15 \mathrm{~s}$ \\
\hline II & (c) $15 \mathrm{~s}$ & (b) $35 \mathrm{~s}$ & (a) $15 \mathrm{~s}$ & $50 \mathrm{~s}$ & $15 \mathrm{~s}$ \\
\hline III & (b) $50 \mathrm{~s}$ & (d) $15 \mathrm{~s}$ & n/a & $50 \mathrm{~s}$ & $15 \mathrm{~s}$ \\
\hline IV & (d) $15 \mathrm{~s}$ & (b) $50 \mathrm{~s}$ & n/a & $50 \mathrm{~s}$ & $15 \mathrm{~s}$ \\
\hline
\end{tabular}

Observe that when maximizing the length and the width of the green waves in both directions for the arterial, then it is not relevant, whether Phase (d) is scheduled immediately before or after Phase (b). The only difference would arise with respect to the other street which is crossing our major road axis. Hence, without loss of generality, we will not distinguish between Sequences III and IV, but only consider Sequence III.

Our goal for the optimization model is thus to select the most appropriate among the Sequences I, II and III for each node along the major road axis in order to maximize the length and the width of the green waves in both directions.

Prior to getting into the details of the optimization model, let us collect several simplifying assumptions which we decided to accept in our model:

- We do not count for any intermediate times for the changes from one phase to the next during operations (e.g. yellow, all red). Yet, we assume this to be negligible, in particular by dedicating the last, say, five seconds of each phase to this purpose.

- We do not consider time losses due to acceleration or deceleration. At first sight, this is no problem, because we are not counting any durations of waiting times in our objective function. Yet, driving from junction $j$ to the next junction $j+1$ will take a few seconds longer for a car that had to stand at red at junction $j$. Moreover, it is beyond the modeling scope of our contribution that cars which have to accelerate after they had to wait at red at junction $j$ risk to prevent cars that arrive from the previous junction $j-1$ (in particular in the case of an ideal green wave) from continuing without any (slight) deceleration. 
- Apart from prioritizing some first seconds of a platoon, we are essentially assuming the traffic flow to be spread equally over the full length of a green phase.

- In particular, when considering the first few seconds of a green phase at junction $j$ as being prioritized when arriving at the next junction $j+1$, these may fit into the green phase there - however, in further downstream considerations their platoon does not need to be part of the prioritized part along the next edge leading to junction $j+2$.

\section{Optimization Model}

We subdivide the presentation of the optimization model into three parts. First, we introduce the basic notation that we are about to use in the sequel. Second, we present the basic structure to compute consistent time values in particular for the points in time of the begin of the green phases (node potentials) and for the time durations e.g. between two junctions or the two opposite directions (periodic tensions). This basic structure will be essentially based on the Periodic Event Scheduling Problem (PESP) as it had been introduced by Serafini and Ukovich (1989), also already in the context of modeling traffic light signals, and applied for instance by Wünsch (2008).

Hereafter, we are getting to the point to measure certain time durations: If a green phase starts a certain number of seconds before or after the upstream platoon arrives, what part of the platoon can pass the function without any stop? Quantifying this objective turns out to yield more complex shapes of the objective function that the linear one that is typically applied to periodic tensions when optimizing over a PESP structure.

Finally, we conclude this section with a short sketch of some alternative integer linear programming model, but whose computational performance turned out to be much worse than that of the model that we are right about to present here.

\section{Notation of Sets and Input Parameters}

We are considering an ordered set $J=(1,2, \ldots,|J|)$ of junctions along the arterial we are applying our optimization model to. The two directions of the arterial are $D=\{W E, E W\}$, where driving from node $j$ to $j+1$ shall be from west to east.

The driving time from junction $i$ to junction $j$, with either $j=i+1$ in the direction WE or $j=i-1$ in the direction $\mathrm{EW}$ is denoted by $d_{i j}$. Next, we are considering the duration of the green phase along the arterial. We assume this to be the same for both directions (see Table 1).

Sometimes we want to put focus on a few first seconds of a green phase, which shall be scheduled preferably without any stop at the next junction with a certain priority. Hence, we introduce a priority parameter $q$ for the duration of either the entire green phase ( $q=$ "no") or just the first prioritized seconds ( $q=$ "yes"). To summarize, we denote the green duration at junction $\mathrm{j}$ with $g d_{i q .}$ In addition, $T$ represents the cycle time that applies to the entire system (e.g. 90 s in the example of Table 1).

Finally, at each node $j$, we are considering the offset between the possible beginning of the green phase in direction WE and the one in direction EW. We denote this amount of offset, or shift, with $s_{j}$. Hence, in the case of Table 1 we find $s_{j}=15 \mathrm{~s}$, and thus the green phases of the two relations may be shifted against each other by either $-15 \mathrm{~s}, 0 \mathrm{~s}$, or $15 \mathrm{~s}$.

\section{Basic Structure of Variables and Essential Constraints}

The periodic events that we are dealing with are either the starting points of a green phase at some junction $j$ into some direction, or the points in time when the platoon from the previous upstream junction starts arriving at junction $j$. Hence, the set $V$ of events - or nodes in a graph model - contains two different kinds of beginnings $B$ : green begin nodes GB and 
arrival begin nodes $A B$. The actual solution of the optimization model are values of the following node variables

$$
\pi_{j, d, b} \in[0, \mathrm{~T}), j \in J, d \in D \text { and } b \in B .
$$

Notice that the two combinations

- $j=1, d=W E$ and $B=A B$

- $j=|J|, d=E W$ and $B=A B$

are of no relevance, because the arriving times of the platoons at the first node in their respective direction is beyond the scope of our considerations.

There will be three types of time durations - or activities, or arcs in a graph model - that we are about to consider:

- driving activities $A_{d}$, $a=\left(v_{1}, v_{2}\right) \in A_{d}$, where $v_{1}=(j, \mathrm{WE}, \mathrm{GB})$ and $v_{2}=(j+1, \mathrm{WE}, \mathrm{AB})$ or $v_{1}=(j, \mathrm{EW}, \mathrm{GB})$ and $v_{2}=(j-1, \mathrm{EW}, \mathrm{AB})$,

- offset (shift) activities $A_{s}$, $a=\left(v_{1}, v_{2}\right) \in A_{s}$, where $v_{1}=(j, \mathrm{WE}, \mathrm{GB})$ and $v_{2}=(j, \mathrm{EW}, \mathrm{GB})$,

- activities $A_{w}$, at the junctions, where cars could face waiting times in case of a stop, $a=\left(v_{1}, v_{2}\right) \in A_{w}$, where $v_{1}=(j, d, \mathrm{AB})$ and $v_{2}=(j, d, \mathrm{~GB})$.

We refer the reader to Figure 4 for an illustration of the interplay between vertices and $\operatorname{arcs} A=A_{d} \cup A_{s} \cup A_{w}$.

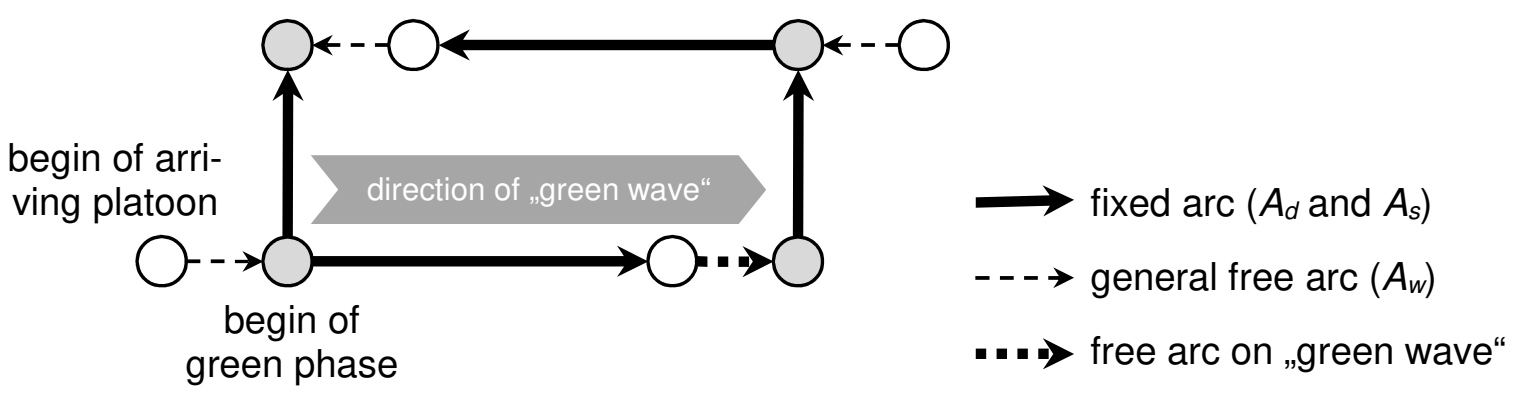

Figure 4 Highlighting the arcs with possible slack along an arterial

As it is well-established when working with Periodic Event Scheduling Problem (PESP) based optimization models, for each arc $a=\left(v_{1}, v_{2}\right) \in A$ we start by considering the pure time difference (aperiodic tension) between the two events $v_{1}$ and $v_{2}$ and denote it by

$$
y_{a}=\pi_{v 2}-\pi_{v 1},
$$

see also (6.26) in Liebchen (2006).

In order to cope with the periodicity (or cyclic structure) of the system, i.e. in particular to avoid computing with negative time differences, for each arc $a \in A$ we introduce periodic tension variables $x_{a}$ for which we define some appropriate lower bound $\ell_{a} \in[0, T)$ and upper bound $u_{a} \in\left[\ell_{a}, \ell_{a}+T\right)$ and require

$$
x_{a} \in\left[\ell_{a}, u_{a}\right] .
$$


The connection between the aperiodic tension variables $y_{a}$ and the periodic tension variables $x_{a}$ is finally established by adding some appropriate integer multiple $p_{a}$ of the cycle time $T$,

$$
x_{a}=y_{a}+T \cdot p_{a} . \text { for all } a \in A,
$$

see also (6.30) in Liebchen (2006). The lower and upper bounds are defined according to Table 2.

Table 2 Lower and upper bounds fort he periodic tension variables

\begin{tabular}{|l|l|l|}
\hline & $\ell_{a}$ & $u_{a}$ \\
\hline$A_{d}$ (driving activity from junction i to $\left.\mathrm{j}\right)$ & $d_{i j}$ & $d_{i j}$ \\
\hline$A_{w}$ & 0 & $\mathrm{~T}-1$ \\
\hline
\end{tabular}

Since we are only allowing for particular offsets (shifts) in order to incorporate aspects of phase selection and phase sequencing, the situation for the arc set $A_{s}$ is slightly more complicated. Yet, there are two possible ways of modeling shifts of $s_{j}$ between the beginnings of the green phases of the two directions at some junction $j$.

Let us first recall a pure PESP-based way, i.e., by involving disjoint intervals. In order to keep only the values $\left\{0, s_{j}, T\right.$-s $\}$ feasible for $x_{a}$, we may replace the arc $a \in A_{s}$ with three parallel copies showing feasible intervals of $\left[0, T-s_{j}\right],\left[s_{j}, T\right]$, and $\left[T-s_{j}, T+s_{j}\right]$, see also $(6.13)$ in Liebchen (2006).

Alternatively, we may enrich the general PESP structure by adding dedicated integer variables and constraints. Actually, the possible time differences between the beginnings of the green phases of the two directions at the same junction $j$ are $\left\{-s_{j}, 0,+s_{j}\right\}$. By introducing a variable $c_{j} \in\{-1,0,+1\}$, the expression $c_{j} \cdot s_{j}$ immediately provides these three desired possible time differences. Yet, it turns out that our scalings in Equations (1) and (3), respectively, prevent us from simply requiring either $y_{a}=c_{j} \cdot s_{j}$ or $x_{a}=c_{j \cdot} \cdot s_{j}$. Instead, we introduce some artificial integer multiplier $r_{j} \in\{0,1\}$ in order to turn the value $-s_{j}$ into $T$-sj, i.e.

$$
x_{a}=c_{j} \cdot s_{j}+T \cdot r_{j}
$$

for all $j \in J$ and $a \in A_{s}$ being the periodic offset/shift tension variable of junction $j$.

To summarize, we set up a PESP-based model to compute points in time for each node and direction along the arterial, at which the respective green phases are scheduled to start. This comes together with computing the appropriate time durations that relate these points in time, namely by directed driving activities between two adjacent junctions, offset (shift) activities between the two directions within the same junction, and activities $A_{w}$ with possible waiting times along one direction at some junction.

It thus remains to weigh the periodic tensions on the arcs $A_{w}$ by some appropriate objective function.

\section{Objective Function Including Further Constraints}

Inspired by the so-called coordination measure of the HBS (FGSV, 2015), we are pursuing the following optimization goal: For each platoon that arrives at some junction, maximize the portion of it that can immediately continue without any stop.

Yet, we are aware that the distribution of the cars in an arriving platoon might not be equal. In contrast, in particular those cars, which just turned from some other crossing street into the arterial will have to arrive at the next junction at red, given that the two junctions are coordinated ideally. Hence, in general we expect the first seconds of a green phase to 
contain relatively more cars than later parts of it. This is why we are putting particular focus on coordinating the traffic signals such that the first few seconds of an arriving platoon will pass the next junction downstream at green, i.e., without any stop. Luckily, it will turn out that a very much similar computation will model both, coordinating the entire green phases for the complete platoons as well as only the first few seconds of an arriving platoon.

The model that we are right about to propose is a piecewise linear convex objective function on the arcs of $A_{w}$, which we want to maximize. This will, of course, require artificial binary variables in order to separate the different linear parts of the objective function from each other.

Let us start by considering the full lengths of the arriving platoons and of the green phases (i.e. $q="$ "no") in the case where

$$
g d_{i, n o} \leq \frac{T}{2}
$$

We present the objective function in Figure 5 and motivate its shape hereafter. The $x$-axis represents the value of $x_{a} \in[0, T), a \in A_{w}$, and we display the value of the objective function on the $y$-axis for the example values $g d=30 \mathrm{~s}$ and $T=90 \mathrm{~s}$.

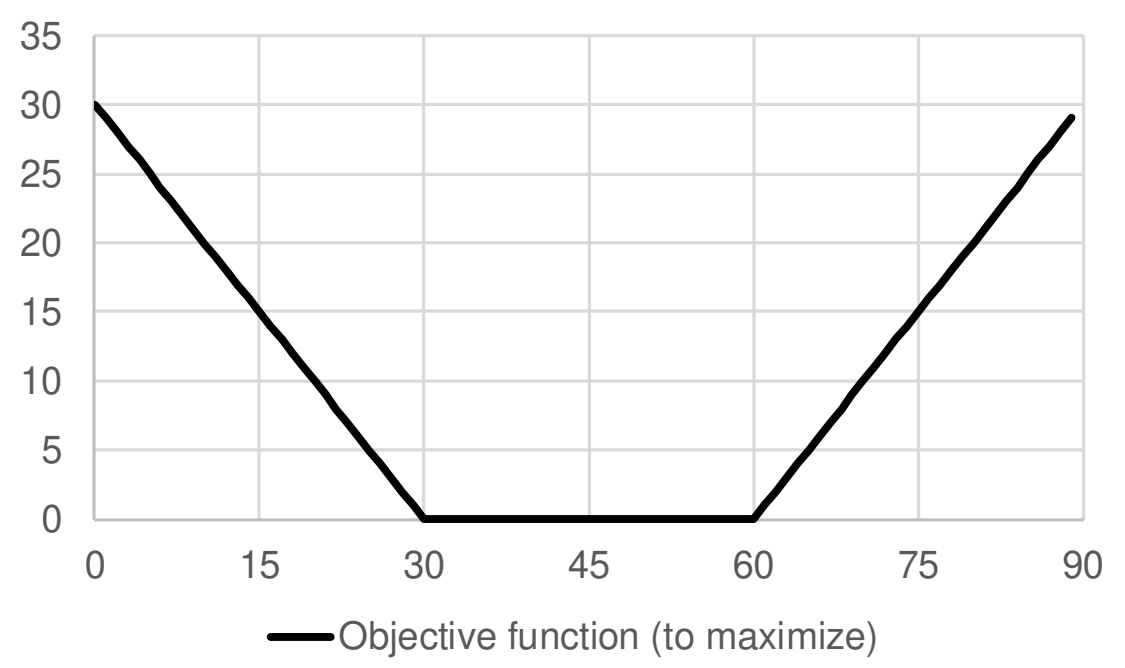

Figure 5 Shape of the piecewise linear convex objective function that we want to maximize in the case of $g d \leq T / 2$

The value $x_{a}=0$ means that there is no time difference between the beginning of the arriving platoon and the beginning of the green phase at this junction. Hence, this is the only relative shift of these two events to each other, where the entire arriving platoon - having length $g d=30$ s - can continue immediately without any stop. Hence, the objective value has to count the full value of $g d-$ here 30 s).

Any offset either to the front or to the end will decrease the number of seconds of the arriving platoon that may continue without any stop accordingly. Hence, when following the objective function from 0 to $g d$ or from $T-1$ to $T$-gd, it decreases with slope one. Finally, if the offset from the beginning of the arriving platoon to the beginning of the green phase is between $g d$ and $T$-gd seconds (recall that we are first considering the case $T \geq 2 g d$ here), then no part of the arriving platoon can continue without stop, such that the objective function has to remain at value zero.

In order to implement this objective function accordingly in the mathematical program, we introduce two binary variables $b_{1}$ and $b_{2}$ : 


$$
\begin{aligned}
& b_{1}=1 \quad \Leftrightarrow \quad x_{a} \geq g d_{i, n o} \\
& b_{2}=1 \quad \Leftrightarrow \quad x_{a} \geq T-g d_{i, n o} .
\end{aligned}
$$

This behavior is obtained by the following two pairs of constraints:

$$
\begin{gathered}
x_{a} \leq \quad g d_{i, n o}+T \cdot b_{1} \\
x_{a} \geq g d_{i, n o}-T \cdot\left(1-b_{1}\right) \\
x_{a} \leq \quad T-g d_{i, n o}+T \cdot b_{2} \\
x_{a} \geq T-g d_{i, n o}-T \cdot\left(1-b_{2}\right)
\end{gathered}
$$

Observe that the value $T$ is playing the role of a "big M value" in order to (de-) activate the respective constraints depending on the actual value of $b_{1}$ and $b_{2}$. Finally, by introducing a variable $z_{a, n o}$ to model the value of the objective function, i.e., the $y$-value in Figure 5 , divided into its three linear parts,

$$
\begin{array}{llc}
z_{a, n o} & \leq & g d_{i, n o}-x_{a}+T \cdot b_{1} \\
z_{a, n o} & \leq & T \cdot\left(b_{2}+\left(1-b_{1}\right)\right) \\
z_{a, n o} & \leq & g d_{i, n o}-T+x_{a}+T \cdot\left(1-b_{2}\right)
\end{array}
$$

our objective function simply turns out to be

$$
\max \sum_{a \in A_{w}} z_{a, n o}
$$

Now, it remains to adapt the definition of the variables $b$ and $z$ to the case of $q=$ "no" but $g d \in[T / 2, T)$, as well as to $q=$ "yes". In the former, the shape of the objective function looks as it is displayed in Figure 6 for the case of $g d=50 \mathrm{~s}$ and $T=90 \mathrm{~s}$.

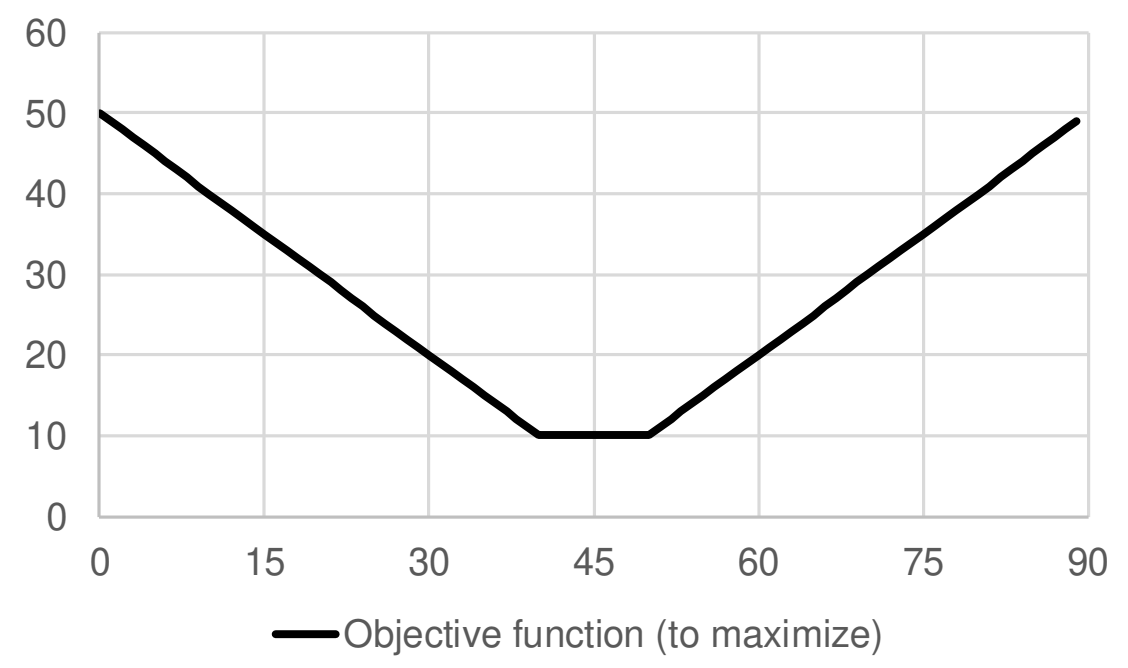

Figure 6 Shape of the piecewise linear convex objective function that we want to maximize in the case of $g d \geq T / 2$

The left gluing point of this piecewise linear function is thus $T$-gd, while the right gluing point is just $g d$. This is motivated by the fact that in the case where $T<2 g d$, there is always a nonempty intersection of the interval of the arriving platoon with the interval of the green phase. Hence, this variant of our objective function never touches the $z_{a, n o}=0$ base line.

In addition to simply exchanging the roles of $g d$ and $T$-gd in (7)-(9), let us state the adjusted version of (10) in its complete form:

$$
\begin{array}{ccc}
z_{a, n o} & \leq & g d_{i, n o}-x_{a}+T \cdot b_{1} \\
z_{a, n o} & \leq & 2 \cdot g d_{i, n o}-T+T \cdot\left(b_{2}+\left(1-b_{1}\right)\right) \\
z_{a, n o} & \leq & g d_{i, n o}-T+x_{a}+T \cdot\left(1-b_{2}\right)
\end{array}
$$


Now, let us turn to the prioritized case, i.e. q="yes". Here, it is in general reasonable to make the following two assumptions:

$$
\begin{aligned}
g d_{i, y e s} & <g d_{i, n o} \\
g d_{i, y e s} & <\frac{T}{2} .
\end{aligned}
$$

A key difference from the case $q=$ "no" lies in the fact that the prioritized part of an arriving platoon shall typically be smaller than the width of the green phase. Hence, the objective function takes a slightly different shape: In the interval $\left[T-\left(g d_{i, n o}-g d_{i, y e s}\right), T\right)$ it takes a plateau where $z_{a, y e s}=g d_{i, y e s}$, because if the wider green phase starts slightly before the (prioritized part of the) arriving platoon arrives, then the entire prioritizes part of the arriving platoon may pass the junction without any stop.

To set up the objective function variable $z_{a, y e s}$ accordingly, we simply introduce a third artificial binary variable $b_{3}$ with $b_{3}=1 \Leftrightarrow x_{2} \geq T$ - $\left(g d_{i, n o}-g d_{i, y e s}\right)$ and finally replace the last inequality in (10) and add one for the last interval, where $z_{a, \text { yes }}=g d_{i, y e s}$ :

$$
\begin{array}{ccc}
z_{a, y e s} & \leq & g d_{i, n o}-T+x_{a}+T \cdot\left(b_{3}+\left(1-b_{2}\right)\right) \\
z_{a, y e s} & \leq & g d_{i, y e s}+T \cdot\left(1-b_{3}\right)
\end{array}
$$

\section{Discussion of an alternative model}

We also implemented an alternative integer linear program to solve the practical problem. In order to measure the part of the arriving platoon that can continue without stop, we were intersecting the periodic interval of the time when a platoon arrives at a junction with the periodic interval that models the green phase at this junction for the appropriate direction, see the appendix for an illustration of this procedure.

Yet, translating this idea into variables and constraints turned out to be somehow inconvenient, also because there can be up to three disjoint fractions of the basic interval $[0, T)$ that constitute the intersection of the two periodic intervals. Apart from the resulting modeling efforts, the performance actually turned out to be totally poor: In the most flexible settings, where the PESP based model that we presented above was able to prove optimality within less than one minute for our test set, the alternative model left a gap of more than ten percent even after a computation time of eight hours.

\section{Some Theoretical Consideration}

Recall the setting of two adjacent junctions as it is displayed in Figure 4. We will show that w.l.o.g. one may require $x_{a}=0$ e.g. in the direction WE, hereby not losing optimality w.r.t. the objective function (11) in the case of solely considering q="no". Putting this building block again and again after itself then enables us to find out that an ideal green wave into one direction is not in conflict with the overall maximum objective value for both directions.

Observe that we are considering the unweighted, thus, most balanced case, in which priority is put neither on one of the two directions, nor on particular junctions ("all equal case"). The phenomenon is somehow similar to the well-known property of linear optimization problems, in which an optimum solution can always be found among the basic solution, which push as many variables as possible to their bounds.

Consider some general optimum solution to the graph in Figure 4. Let us denote by $X_{W E}$ and $x_{\mathrm{EW}}$ the periodic tension values of the two waiting arcs $a_{W E}, a_{\mathrm{EW}} \in A_{w}$. Together with four nonwaiting arcs, these form an oriented circuit. From the general theory of periodic tensions, it is 
well-known (see e.g. Liebchen, 2006) that there is some integer $k \in \mathbf{Z}$ such that the following cycle equation (15) is satisfied

$$
x_{W E}+x_{E W}+\sum_{a \in A_{d}} x_{a}+x_{s, 2}-x_{s, 1}=k \cdot T,
$$

where $x_{s, j}$ denotes the shift (offset) variable at junction $j$.

Except for $x_{W E}$ and $x_{E W}$, we assume the other periodic tension variables to be fixed to the values that they take in the optimum solution that we are starting with. Then, let us motivate why we may push any slack from $X_{W E}$ to $X_{E W}$, hereby not increasing the objective value.

Let us consider the following six cases:

(a) $g d \leq T / 2$ and $x_{W E} \in[0, g d]$

In order to obtain $X_{W E}=0$, being the unique value that leads to the maximum objective value in the case of $q=$ "no", we shift the system by an amount of $x_{\text {WE. }}$ On the waiting arc in direction WE, in this case, this increases the objective value by exactly $X_{W E}$. In turn, the decrease of the objective value on the arC $X_{E W}$ cannot exceed this increase, because the absolute value of the slope in Figure 6 is bounded from above by one. Hence, we come up with a signal schedule of at least the same objective value, but now showing $X_{W E}=0$.

(b) $g d \leq T / 2$ and $x_{W E} \in(g d, T-g d)$

Here, we start with the smallest possible objective value for $X_{W E}$, and by setting $x_{W E}=0$ immediately, we realize the maximum possible increase in the objective function displayed in Figure 6 . But since the increase is bounded from above by $g d_{i, \text { no }}$, so is the decrease of the possible contribution of $x_{\mathrm{EW}}$ to the objective function. Again, we come up with a signal schedule of at least the same objective value, but having $X_{W E}=0$.

(c) $g d \leq T / 2$ and $x_{W E} \in[T-g d, T)$

This case is most similar to Case (a), but here, we just shift the system by an amount of T- $X_{W E}$. Doing so, every unit of shift increases the objective value that we associate with $X_{W E}$ by one, while the decrease that we face on arc $X_{E W}$ is again bounded from above by $\mathrm{T}-\mathrm{X}_{\mathrm{WE}}$, due to the property that the absolute value of the slope is at most one.

(d) $\quad g d>T / 2$

The three detailed cases $X_{\mathrm{WE}} \in[0, T-g d], X_{\mathrm{WE}} \in[T-g d, g d]$, and $x_{\mathrm{WE}} \in[g d, T)$ are in fact the counterparts of Cases (a), (b), and (c), respectively. There, the very same reasoning applies.

We summarize the previous considerations in the following theorem.

Theorem 1 Given a bidirectional road arterial with $|\mathrm{J}|$ junctions, common cycle time $T$ and identical green durations $g d$ in both directions and at each of the junctions. Consider as objective function the sum of the following term over both directions and all junctions: Maximize the portion of each platoon that arrives at junction $j$ and which can pass junction $j$ immediately at green without having to stop, i.e., without any priorities among the junctions or directions. Then, there is always an optimum solution that establishes an ideal green wave into one direction, i.e., where at each junction the entire arriving platoon of that direction can pass without any stop. 


\section{Data Set}

Recall from the introduction that it is trivial to establish green waves into both opposite directions, as long as the driving times between two adjacent junctions is an integer multiple of half the cycle time $T$. In order to demonstrate the potential of the proposed integer linear optimization model, of course we avoid these well-known ideal driving times.

In this spirit, consider the driving times along the artificial road arterial with a common cycle time $T$ of $T=90$ s that is shown in Figure 7 , and which is going to serve as input for our computational results in the next section.

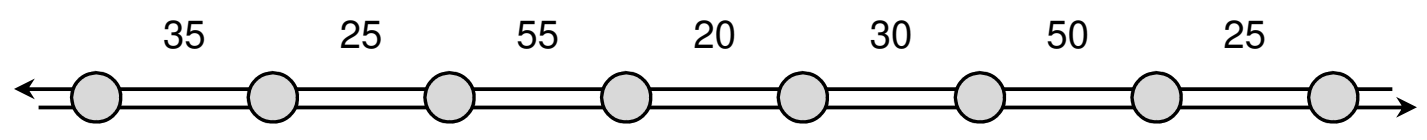

Figure 7 Visualization of the bi-directional arterial on which we perform our computations. The numbers in between two subsequent junctions denote the driving times (in seconds) between them.

In addition to avoiding immediately integer multiples of half the period time between any two adjacent junctions, the driving times in Figure 7 mainly also keep this property when considering sequences of two or even three road segments, as can be observed in Table 3.

\begin{tabular}{|l|r|r|r|r|r|r|r|}
\hline from $\backslash$ to & 2 & 3 & 4 & 5 & 6 & 7 & 8 \\
\hline 1 & 35 & 60 & 115 & 135 & 165 & 215 & 240 \\
\hline 2 & & 25 & 80 & 100 & 130 & 180 & 205 \\
\hline 3 & & & 55 & 75 & 105 & 155 & 180 \\
\hline 4 & & & & 20 & 50 & 100 & 125 \\
\hline 5 & & & & & 30 & 80 & 105 \\
\hline 6 & & & & & & 50 & 75 \\
\hline 7 & & & & & & & 25 \\
\hline
\end{tabular}

Table 3 Partial sums of the driving times along the road arterial (displayed only from west to east, due to symmetry)

Notice that only the partial sums from 1 to 5 , from 2 to 7 , and from 3 to 8 constitute exact multiples of half the cycle time, i.e., of $45 \mathrm{~s}$ in this data set. Moreover, only the partial sums from 2 to 6 and from 4 to 6 and from 6 to 7 differ from an integer multiple of half the cycle time by at most five seconds.

In our computations it will turn out that if at each junction the times of the green phases of both directions are precisely the same, i.e. $c_{j}=0$, then the quality of the coordination has to remain rather limited.

\section{Computational Results}

We report the fraction (in percent) of

$$
\sum_{d \in\{W E, E W\}} \sum_{j \in J} g d_{j, q}, q \in\{\text { yes, no }\}
$$

which our objective function (11) is able to attain as the central quality measure. In the computations that we perform, we vary in particular

- the length of the prioritized green duration $g d_{j, y e s}$,

- the degree of freedom whether both directions must have green at the very same time (i.e. $c_{j}=0$ ), whether the same pattern $c_{j}$ shall apply to each junction, or if we may 
select the phase sequence at each junction independently from any other junction (i.e. $c \in\{-1,0,+1\}$ ),

- the requirement whether an ideal green wave into one direction (w.I.o.g. WE) has to be established (i.e. $x_{a}=0$, for $a \in A_{w}$ in direction $d=W E$ ),

- and finally global objective weights by which the sum over $q=y e s$ and $q=$ no may we weighted by either one or ten.

The computational results that are reported in Table 4 have been obtained on an Intel Core i5-5200U at $2.20 \mathrm{GHz}$ with $8 \mathrm{~GB}$ RAM, where the model was implemented in AIMMS 4.79.4.17 and finally solved with CPLEX 20.1.

\begin{tabular}{|c|c|c|c|c|c|c|c|c|}
\hline ID & $g d_{j, y e s}$ & $\begin{array}{l}\text { objective } \\
\text { weight for } \\
q=\text { yes }\end{array}$ & $\begin{array}{l}\text { objective } \\
\text { weight } \\
\text { for } q=\text { no }\end{array}$ & $\begin{array}{l}\text { sequence } \\
\text { require- } \\
\text { ment }\end{array}$ & $\begin{array}{l}\text { green } \\
\text { wave } \\
\text { WE? }\end{array}$ & $\begin{array}{l}\text { comp. } \\
\text { time } \\
\text { (s) }\end{array}$ & $\begin{array}{l}\text { solution } \\
\text { quality for } \\
q=\text { yes }\end{array}$ & $\begin{array}{l}\text { solution } \\
\text { quality for } \\
q=\text { no }\end{array}$ \\
\hline & 10 & & & reference & & & $140(100 \%)$ & $700(100 \%)$ \\
\hline 11 & 10 & 1 & 10 & $c_{f}=0$ & yes & 0.1 & $100(71 \%)$ & $500(71 \%)$ \\
\hline 12 & 10 & 1 & 10 & $c_{j}$ equal & yes & 0.1 & $100(71 \%)$ & $500(71 \%)$ \\
\hline 13 & 10 & 1 & 10 & $c_{j} \in\{-1,0,1\}$ & yes & 0.3 & $125(89 \%)$ & $610(87 \%)$ \\
\hline 14 & 10 & 10 & 1 & $c_{j}=0$ & yes & 0.1 & $100(71 \%)$ & $500(71 \%)$ \\
\hline 15 & 10 & 10 & 1 & $c_{j}=0$ & no & 1.3 & $140(100 \%)$ & 400 (57\%) \\
\hline 16 & 10 & 10 & 1 & $c_{j}$ equal & yes & 0.1 & $100(71 \%)$ & $500(71 \%)$ \\
\hline 17 & 10 & 10 & 1 & $c_{j}$ equal & no & 2.5 & $140(100 \%)$ & $400(57 \%)$ \\
\hline 18 & 10 & 10 & 1 & $c_{j} \in\{-1,0,1\}$ & yes & 0.2 & $140(100 \%)$ & $580(83 \%)$ \\
\hline 19 & 10 & 10 & 1 & $c_{j} \in\{-1,0,1\}$ & no & 2.7 & 140 (100\%) & $580(83 \%)$ \\
\hline & 25 & & & reference & & & $350(100 \%)$ & $700(100 \%)$ \\
\hline 21 & 25 & 1 & 10 & $c_{f}=0$ & yes & 0.1 & $240(69 \%)$ & $500(71 \%)$ \\
\hline 22 & 25 & 1 & 10 & $c_{j}$ equal & yes & 0.1 & $240(69 \%)$ & $500(71 \%)$ \\
\hline 23 & 25 & 1 & 10 & $c_{j} \in\{-1,0,1\}$ & yes & 0.3 & $320(91 \%)$ & $610(87 \%)$ \\
\hline 24 & 25 & 10 & 1 & $c_{f}=0$ & yes & 0.1 & $240(69 \%)$ & $500(71 \%)$ \\
\hline 25 & 25 & 10 & 1 & $c_{f}=0$ & no & 2.4 & $320(91 \%)$ & $450(64 \%)$ \\
\hline 26 & 25 & 10 & 1 & $c_{j}$ equal & yes & 0.1 & $240(69 \%)$ & $500(71 \%)$ \\
\hline 27 & 25 & 10 & 1 & $c_{j}$ equal & no & 2.9 & $320(91 \%)$ & $450(64 \%)$ \\
\hline 28 & 25 & 10 & 1 & $c_{j} \in\{-1,0,1\}$ & yes & 1.3 & $340(97 \%)$ & $580(83 \%)$ \\
\hline 29 & 25 & 10 & 1 & $c_{j} \in\{-1,0,1\}$ & no & 0.2 & $350(100 \%)$ & 580 (83\%) \\
\hline
\end{tabular}

Table 4 Input parameter settings and solution quality measures for the computations that we performed on the arterial that is shown in Figure 7

Recall from Theorem 1 that given that we put emphasis on the full width of the green band (i.e. q="no"), then enforcing an ideal green wave into one direction will not decrease the corresponding part of the objective function. This is why we refrain from reporting rows with

- objective weight for $q=y e s$ equal to 1 ,

- objective weight for $q=$ no equal to 10 , and

- green wave WE "no".

In contrast, for instance when comparing the last two rows of Table 4 (i.e. Cases 28 and 29) one may detect a combination where enforcing an ideal green wave into one direction decreases the part of the objective function that is dedicated to the prioritized part of the green band, in this case the first 25s. This effect can also be observed locally in the building block that is shown in Figure 4 when selecting the following values

- cycle time $T=90 \mathrm{~s}$,

- driving time $d_{i j}=d_{j i}=25 \mathrm{~s}$,

- green duration $g d_{j, n_{0}}=50 \mathrm{~s}$, and

- green duration $g d_{j, y e s}=25 \mathrm{~s}$. 
Nota bene that a solution quality value $(q=$ no) of only $50 \%$ would already be attained by simply establishing a unidirectional ideal green wave, while $100 \%$ means ideal green waves into both directions. Hence, an increase from $71 \%$ (no decisions of phase sequencing, Case 11) to $87 \%$ (integrating decisions in particular of phase sequencing) might be considered being significant.

As for the solution quality, it is getting obvious that only when allowing $c_{j} \in\{-1,0,1\}$ the best objective values can be achieved. We illustrate this further by showing the bidirectional timespace diagrams (junctions are represented by vertical lines, integer multiples of the cycle time $T=90$ s by the grey horizontal lines) of the green bands for Cases 11 and 18 here (cf. Figure 8 and Figure 9), and refer the interested reader to the appendix for additional illustrations (Cases 13, 28, and 29).

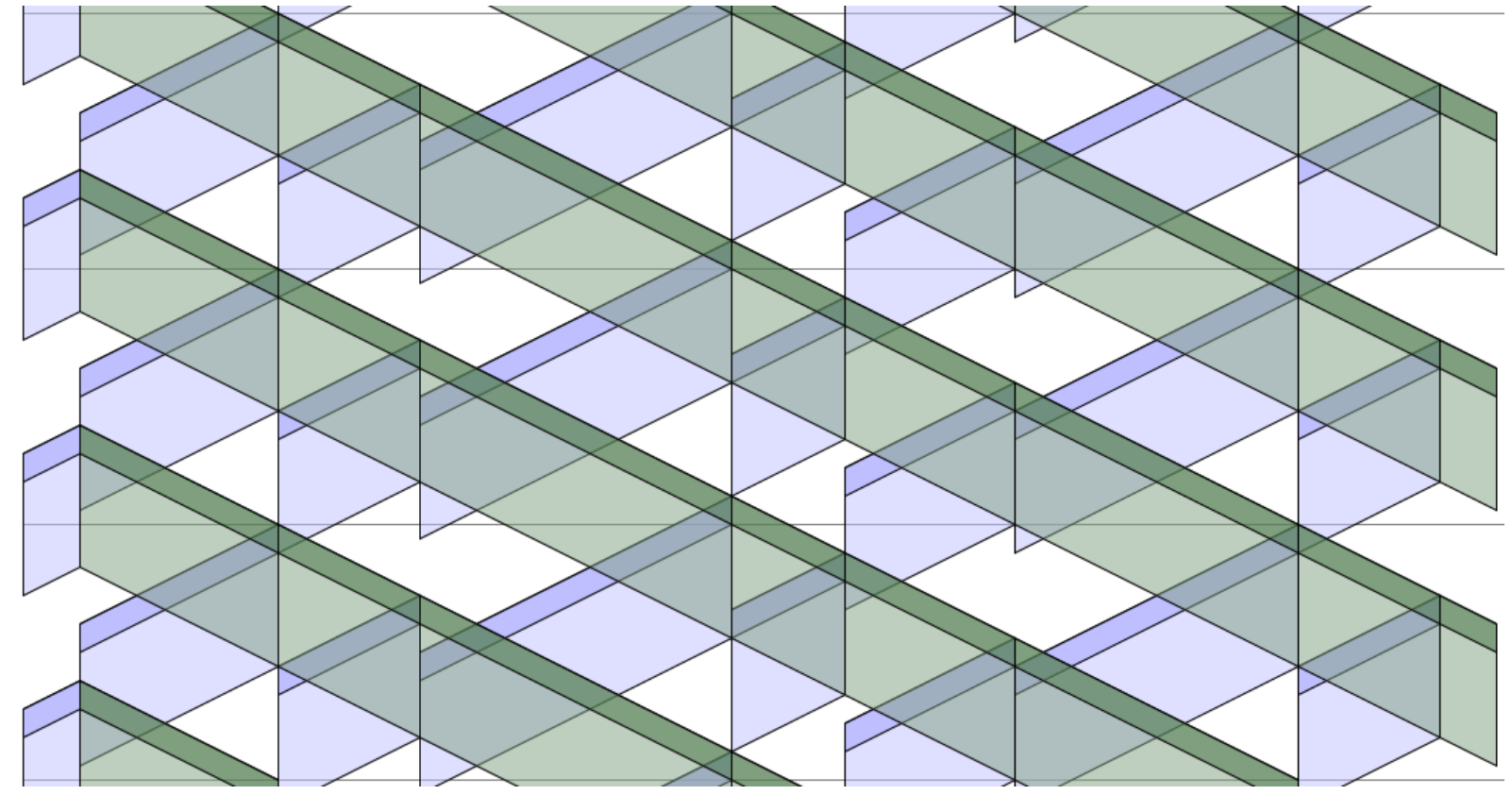

Figure 8 Bidirectional time-space diagrams of the green bands for Case 11: At each junction (horizontal axis), both directions show green always at the same time, and an ideal green wave is established in direction WE. The solution quality for both, $q=y e s$ and $q=$ no is $71 \%$.

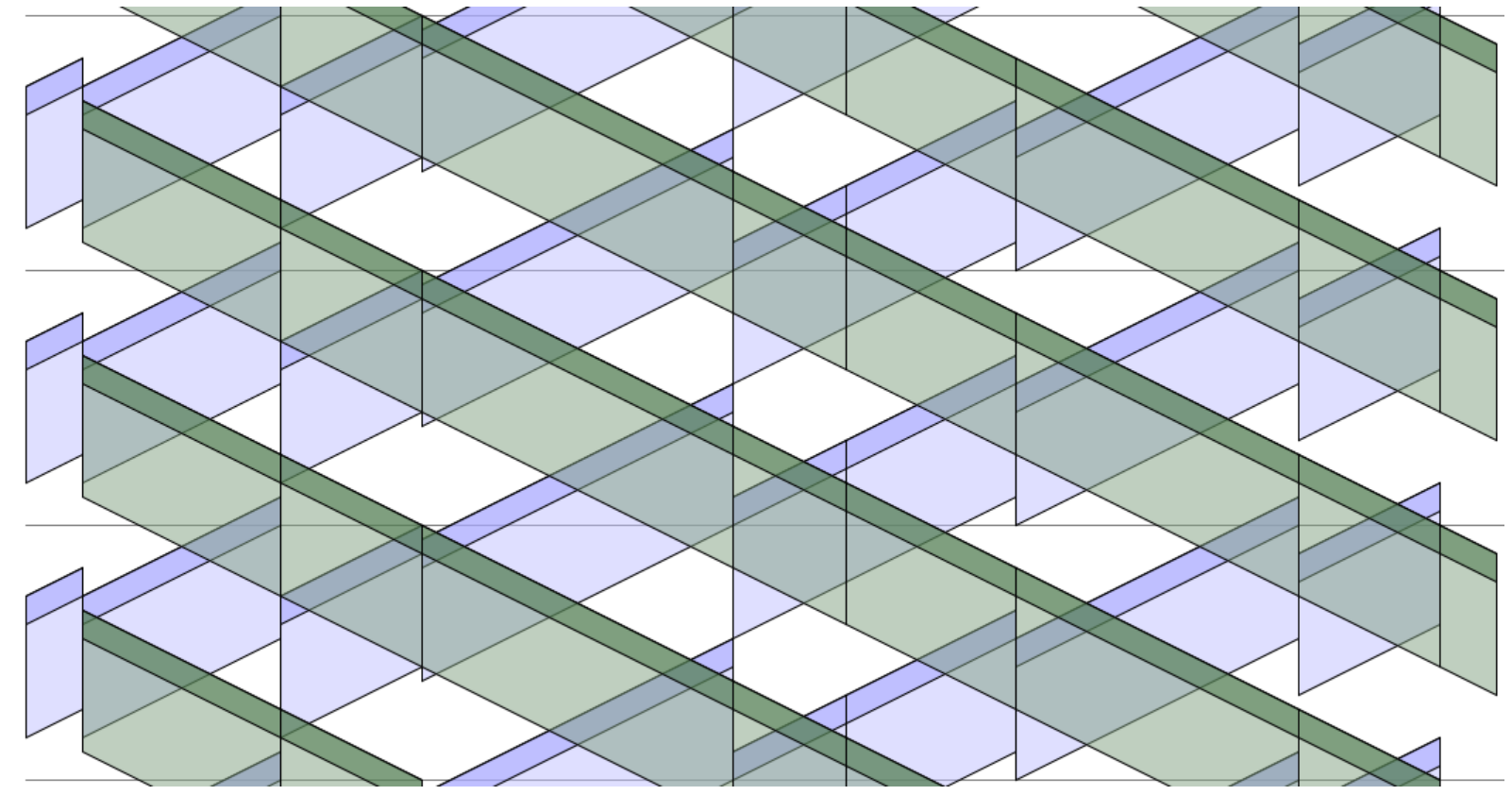


Figure 9 Bidirectional time-space diagrams of the green bands for Case 18: At each junction (horizontal axis), the phase sequence may be selected independently from each other, and an ideal green wave is established in direction WE. The solution quality for $q=n o$ is $83 \%$ (i.e. $83 \%$ of all arriving platoon widths may continue without any stop) and for $q=y e s$ (darker upper parts - 10s - of the green bands of width 50 s each) even $100 \%$.

\section{Conclusions}

We proposed a PESP-based integer linear optimization model that adds decisions of phase sequencing, partly even phase selection, to the task of coordinating traffic lights. We applied this model to an artificial arterial with eight junctions. In our computational results we provide clear evidence for the benefit of including decisions of phase sequencing (phase selection): On our data set, the solution quality in terms of the parts of all arriving platoons which may pass the next junction without any stop increases from $71 \%$ to $87 \%$ (cf. Cases 11 and 13).

From a theoretical perspective, we showed that even in the "all equal case", i.e., where there is no priority between any of the junctions or of the two directions of the arterial, there is always an optimum solution with respect to the maximization of the part of an arriving platoon which can continue at the next junction without any stop, which established an ideal green wave into one direction.

In particular, when comparing our somehow local objective function (11) to the actual coordination measure according to the HBS (FGSV, 2015), one relevant potential line of improvement becomes obvious: turning the local nature of our objective function into one that follows the green band over more than just the immediately next junction - i.e. along (small) paths rather than just edges.

\section{Acknowledgment}

The author thanks Berenike Masing and Niels Lindner for a fruitful discussion which let us finally formulate Theorem 1, and another very useful suggestion by Niels Lindner lead to the way in which the intersection of cyclic intervals can be modeled according to the Appendix. In addition, the author thanks Bernhard Friedrich, Klaus Nökel and Peter Wagner for worthwhile comments.

\section{References}

J. C. Binning, M. R. Crabtree and G. L. Burtenshaw (2010), "TRANSYT 14 User Guide", ISSN 1365-6929

Department of Transport (DOT, 2021), "Traffic Signal Timing Manual, Chapter 6, Coordination", Federal Highway Administration, https://ops.fhwa.dot.gov/publications/fhwahop08024/chapter6.htm (accessed on Oct, $5^{\text {th }}$, 2021)

Myungeun Eom and Byung-In Kim (2020), "The traffic signal control problem for intersections: a review”, European Transport Research Review 12:50

Forschungsgesellschaft für Straßen- und Verkehrswesen (FGSV, 2015), "Handbuch für die Bemessung von Straßenverkehrsanlagen (HBS) - Teil S: Stadtstraßen", FGSV-Verlag GmbH, Köln, 299, ISBN 978-3-8446-103-3

Foresite Group (2021), "A Beginners' Guide to Traffic Signal Timing", https://www.foresitegroup.net/a-beginners-guide-to-signal-timing/ (accessed on Oct, $5^{\text {th }}$, 2021) 
Refael Hassin (1996), "A flow algorithm for network synchronization”, Operations Research, 44(4), 570-579

Christian Liebchen (2006), "Periodic Timetable Optimization in Public Transport", dissertation.de - Verlag im Internet

Paolo Serafini and Walter Ukovich (1989), "A mathematical model for periodic scheduling problems", SIAM Journal on Discrete Mathematics, 2(4), 550-581

Gregor Wünsch (2008), "Coordination of Traffic Signals in Networks", PhD Thesis, Technical University Berlin, Germany

Chao Zhang, Yuanchang Xie, Nathan H. Gartner, Chronis Stamatiadis and Tugba Arsava (2015), "AM-Band: An Asymmetrical Multi-Band model for arterial traffic signal coordination", Transportation Research Part C, 58, 515-531

\section{Appendix}

\section{Intersection of Cyclic Intervals}

The main idea for computing the intersection of two cyclic intervals is to

- first extend any cyclic interval that exceeds the cycle time $T$ onto the non-cyclic interval $[0,2 T)$,

- project any part of the non-cyclic interval $[T, 2 T)$ onto the basic interval $[0, T)$,

- and then finally intersect several smaller non-cyclic subintervals within $[0, T)$.

This process is illustrated in Figure 10. The two bars on top of the time axis indicate two given cyclic input intervals. Think of the light blue interval as the time during which the cars from the previous upstream node are arriving at the present node and denote it by $A=\left[\ell_{A}, U_{A}\right]_{T}$, and of the dark green interval as the green phase at the present node and denote it by $G=\left[\ell_{G}, U_{G}\right] T$. For example, in the top-left subfigure, an ideal synchronization is shown.

The lower bounds $\ell_{A}$ and $\ell_{G}$ of the given cyclic input intervals are scaled to $[0, T)$, thus, sometimes their upper bound may exceed $T$., hence, in this case, we find $u_{A}, u_{G} \in[T, 2 T)$. Below the time axis, the up to two parts of $[0, T)$ per cyclic input interval are displayed, which result from projecting any non-empty part of $\left[T, U_{A}\right]$ and $\left[T, U_{G}\right]$ onto $[0, T)$. Finally, the unfilled bars on the bottom represent the intersection of the two cyclic input intervals after having been scaled to the basic interval $[0, T)$. This may result in one, two or even three subintervals of $[0, T)$.
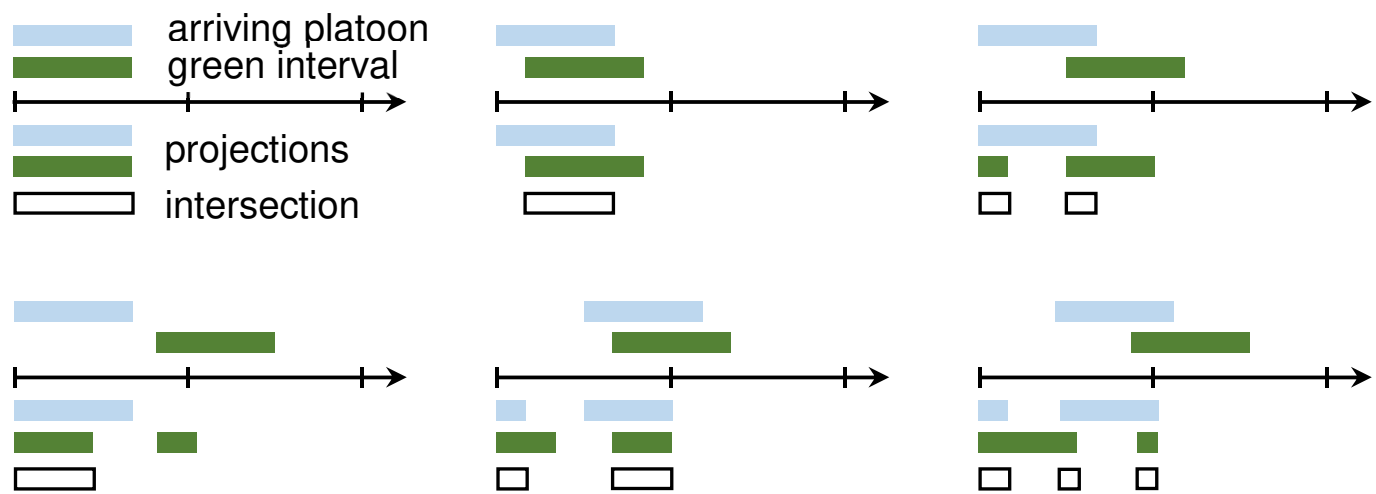
Figure 10 Selected configurations of two cyclic intervals for their intersection

Yet, we refrain from puzzling around with precise notation in order to squeeze this idea into variables and constraints of an integer linear optimization model.

\section{Further Charts}

In the remainder, we provide the time-space diagrams for Cases 13, 28, and 29 in the same design as Figure 8 and Figure 9.

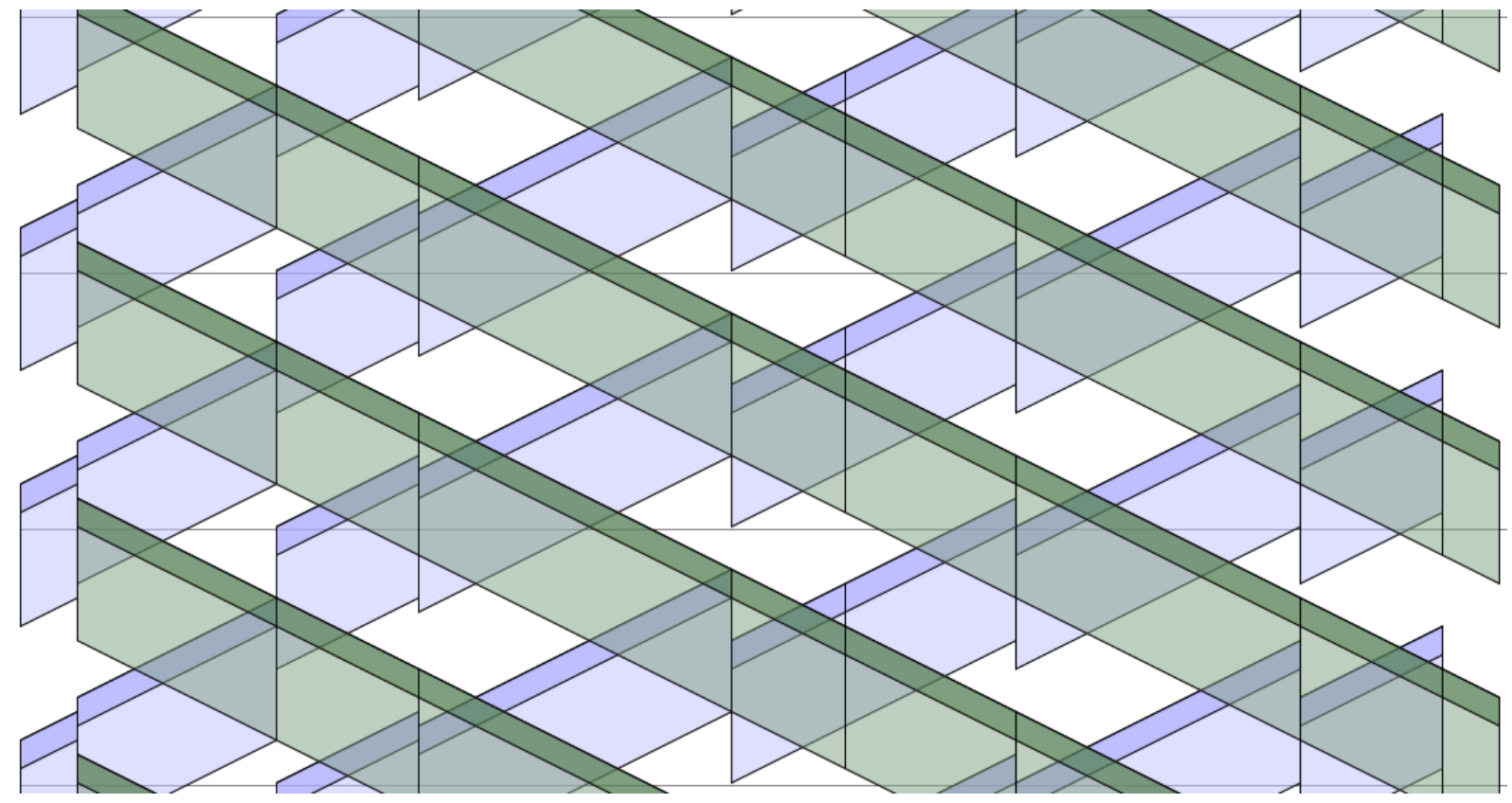

Figure 11 Compare this Case 13 with Case 11 in order to immediately experience the benefit of arranging tailored phase sequences at each individual junction. Here, the solution quality for $q=n o$ is $87 \%$ compared to only $71 \%$ in Case 11, hereby illustrating the benefit of including decisions of phase sequencing (and partly even phase selection) tot he task of coordinating traffic lights along an arterial..

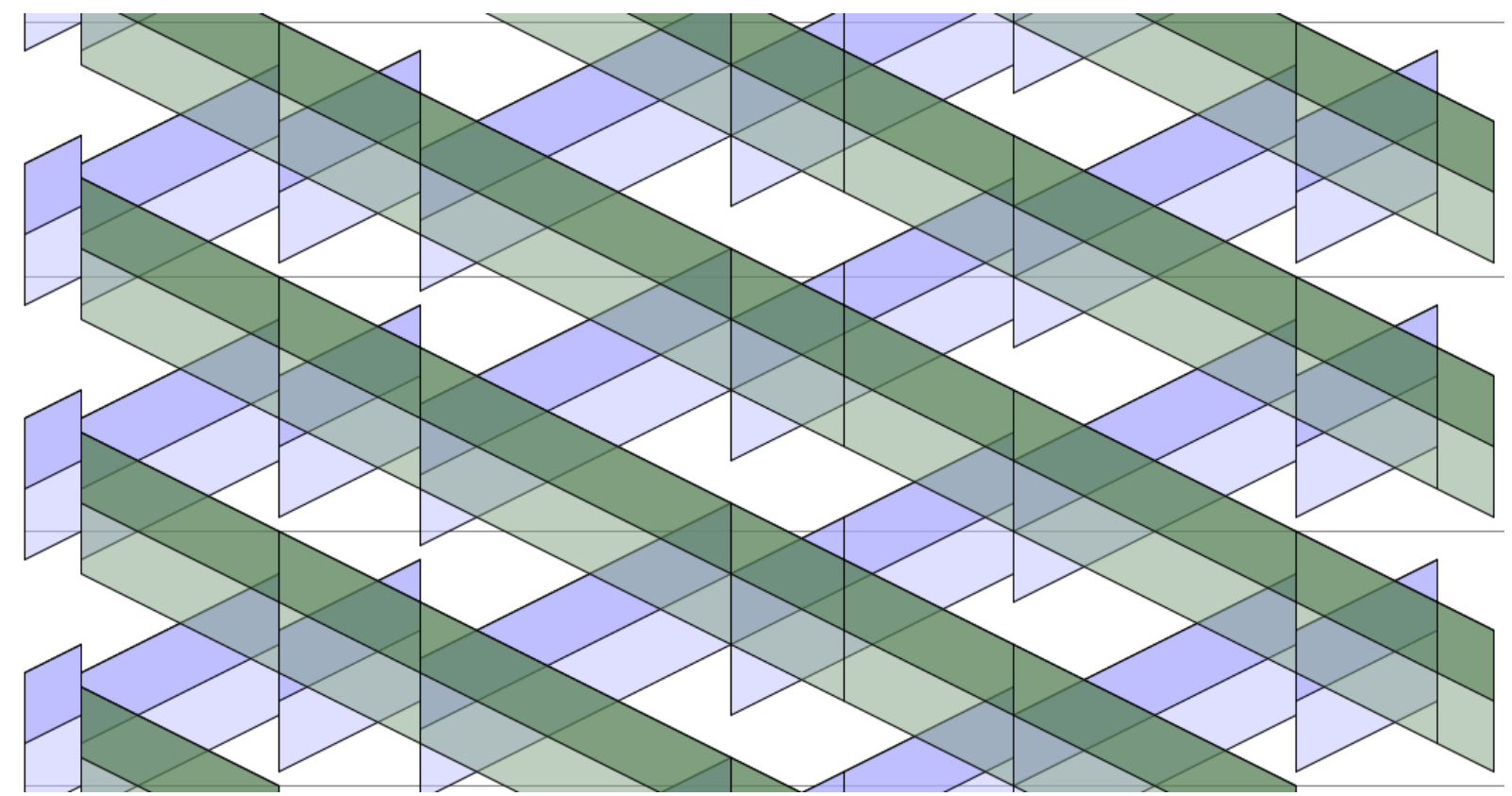

Figure 12 In Case 28, we enforced an ideal green wave in direction WE, but at the third junction (counted from west to east), a smal part of the prioritized $50 \%$ of the arriving platoon faces a stop. 


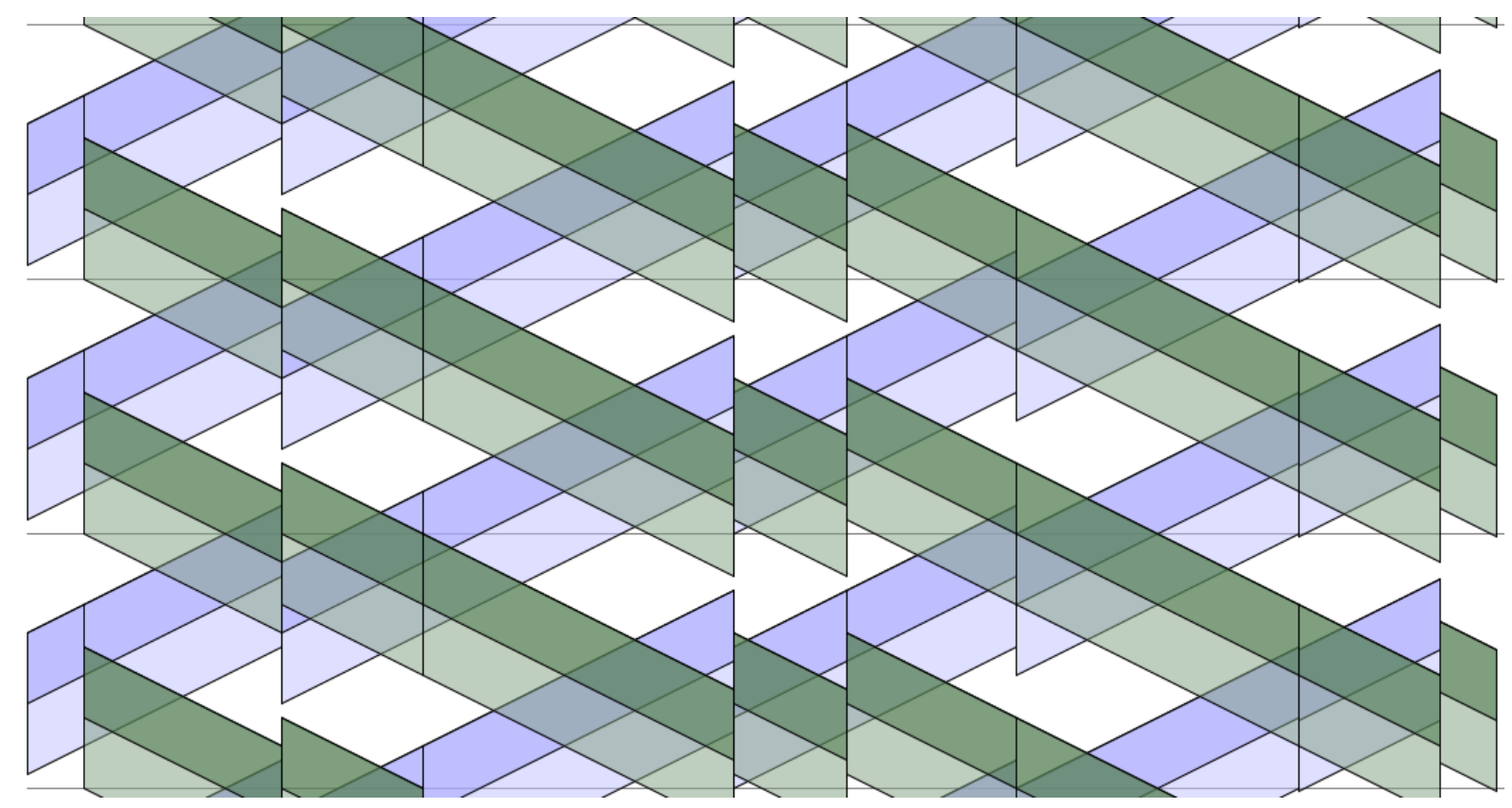

Figure 13 In Case 29, 100\% of the prioritized 50\% of each arriving platoon can continue without any stop at the next junction downstram - but there is no ideal green wave into any of the two directions. 